International Journal of Pure and Applied Mathematics

Volume 109 No. 3 2016, 651-663

ISSN: 1311-8080 (printed version); ISSN: 1314-3395 (on-line version)

url: http://www.ijpam.eu

doi: 10.12732/ijpam.v109i3.13

\title{
SOME FIXED POINT THEOREMS ON THE SUM AND PRODUCT OF OPERATORS IN TENSOR PRODUCT SPACES
}

\author{
Dipankar Das ${ }^{1}$, Nilakshi Goswami ${ }^{2}$ \\ ${ }^{1,2}$ Department of Mathematics \\ Gauhati University \\ Guwahati, 14, Assam, INDIA
}

\begin{abstract}
Let $X$ and $Y$ be Banach spaces and $P$ and $Q$ be two subsets of $X$ and $Y$ respectively. Let $T_{1}: X \otimes_{\gamma} Y \rightarrow X$ and $T_{2}: X \otimes_{\gamma} Y \rightarrow Y$ be two mappings and $S$ be a self mapping on $P \otimes Q$. Using $T_{1}$ and $T_{2}$ we define a self mapping $T$ on $X \otimes_{\gamma} Y$. Different conditions under which $T+T S+S$ has a fixed point in $P \otimes Q$ are established here. Analogous results are also established taking the pair $\left(T_{1}, T_{2}\right)$ as $\left(k, k^{\prime}\right)$ contraction mappings. Again considering $X \otimes_{\gamma} Y$ as a reflexive Banach space. We derive the conditions for $\frac{1}{m}(T+S T+S), m>$ 2, $m \in N$, for having a fixed point in $P \otimes Q$. Some iteration schemes converging to a fixed point of $T+S T+S$ in $P \otimes Q$ are also presented here.
\end{abstract}

AMS Subject Classification: 46B28, 47A80, 47H10

Key Words: projective tensor norm, reflexive tensor product, demiclosed mapping

\section{Introduction}

In 1955, Krasnoselskii [13], proved a fixed point theorem for a sum of two mappings $T$ and $S$ on a non-empty closed convex bounded subset of a Banach space $X$. This theorem was generalized and extended by different researchers, viz., Cain and Nashed [4], Nashed and Wong [14], Edmunds [9], Reinermann [16], Sehgal and Sing [18], Vijayaraju [19], etc., considering $S$ and $T$ as different types

Received:

Revised:

May 5, 2016

August 27, 2016

Published: $\quad$ September 30, 2016 (c) 2016 Academic Publications, Ltd.

url: www.acadpubl.eu 
of mappings. Taking Browder's fixed point theorem, in 1982, Olga Hadzic ( [10], [11]) derived some fixed point theorems for $T+S$ on a Banach space $X$. In 2003, Dhage [5], discussed two fixed point theorems for the sum and product of two operators. In this paper, we consider two Banach spaces $X$ and $Y$ and there projective tensor product $X \otimes_{\gamma} Y$. We consider a pair of mappings $T_{1}: X \otimes_{\gamma} Y \rightarrow X$ and $T_{2}: X \otimes_{\gamma} Y \rightarrow Y$, and from this pair we construct a self mapping $T$ on $X \otimes_{\gamma} Y$. Let $P$ and $Q$ be two subsets of $X$ and $Y$ respectively and $S$ be a self mapping on $P \otimes Q$. We derive some fixed point theorems for $T+T S+S$ in the subset $P \otimes Q$ of $X \otimes_{\gamma} Y$. Some iteration schemes converging to this fixed point will also be discussed here.

\section{Preliminaries}

Definition 2.1. Let $X$ and $Y$ be normed spaces. A mapping $T: X \rightarrow Y$ is called non-expansive if and only if $\|T x-T y\| \leqslant\|x-y\|, \forall x, y \in X$. It is said to be demi-closed if its graph is sequentially closed in the product of the weak topology on $X$ with the norm topology on $Y$ (refer. to $([15])$ ).

A mapping $T: X \rightarrow Y$ is called contraction if and only if

$$
\|T x-T y\| \leqslant r\|x-y\|
$$

where $r$ is real number with $0 \leqslant r<1, \forall x, y \in X$.

Definition 2.2. (see [3]) Let $X$ be a Banach space and $f$ be a continuous (not necessarily linear) mapping of $X$ into itself. The mapping $f$ is said to be completely continuous if the image under $f$ of each bounded set of $X$ is contained in a compact set.

Theorem 2.3. (see [3]) Let $f$ be a completely continuous self mapping on a Banach space $X$. If for some positive integer $m, f^{m}(X)$ is bounded, then $f$ has a fixed point.

Theorem 2.4. (Schauder's Fixed-Point Theorem, see [17]) Let $K$ be a nonempty, convex and compact subset of a normed space. Any continuous mapping $T: K \rightarrow K$ has at least one fixed point.

Theorem 2.5. (Banach's Contraction Mapping Principle, see [1]) Let $(X, d)$ be a complete metric space, $c \in(0,1)$ and $T: X \rightarrow X$ be a mapping such that for each $x, y \in X, d(T x, T y) \leqslant c d(x, y)$. Then $T$ has a unique fixed point $a \in X$, such that for each $x \in X, \lim _{n \rightarrow \infty} T_{n} x=a$. 
Projective Tensor Product 2.7. (see [2]) Given normed spaces $X$ and $Y$, the projective tensor norm $\gamma$ on $X \otimes_{\gamma} Y$ is defined by

$$
\|u\|=\inf \left\{\sum_{i}\left\|x_{i}\right\|\left\|y_{i}\right\|: u=\sum_{i} x_{i} \otimes y_{i}\right\}
$$

where the infimum is taken over all (finite) representations of $u$.

Lemma 2.8. (see [2]) $X \otimes_{\gamma} Y$ can be represented as a linear subset of $B L\left(X^{*}, Y^{*} ; F\right)$ consisting of all elements of the form $u=\sum_{i} x_{i} \otimes y_{i}$ where $\sum_{i}\left\|x_{i}\right\|\left\|y_{i}\right\|<\infty$. Moreover, $\|u\|=\inf \left\{\sum_{i}\left\|x_{i}\right\|\left\|y_{i}\right\|\right\}$ over all such representations of $u$.

Definition 2.9 Let $P$ and $Q$ be bounded subsets of the Banach spaces $X$ and $Y$ respectively. A pair of mappings $T_{1}: P \otimes Q \rightarrow P$ and $T_{2}: P \otimes Q \rightarrow Q$ is called $\left(k, k^{/}\right)$contraction mappings if:

(i) $\left\|T_{1} u-T_{1} v\right\| \leqslant \frac{k}{M_{2}}\|u-v\|, \frac{k}{M_{2}}<1$,

(ii) $\left\|T_{2} u-T_{2} v\right\| \leqslant \frac{k^{\prime}}{M_{1}}\|u-v\|, \frac{k^{\prime}}{M_{1}}<1$,

(iii) $\left\|T_{1} u\right\| \leqslant M_{1},\left\|T_{2} u\right\| \leqslant M_{2} \forall u, v \in P \otimes Q$,

where $P \otimes Q$ is bounded by $M_{1} M_{2}$.

\section{Main Results}

First, we consider $P$ and $Q$ as bounded subsets of the Banach spaces $X$ and $Y$ respectively.

Theorem 3.1. Let $P$ and $Q$ be as defined above and let $T_{1}: X \otimes_{\gamma} Y \rightarrow X$ and $T_{2}: X \otimes_{\gamma} Y \rightarrow Y$ be two continuous mappings such that $T_{1}(P \otimes Q) \subseteq P$ and $T_{2}(P \otimes Q) \subseteq Q$. We define $T: X \otimes_{\gamma} Y \rightarrow X \otimes_{\gamma} Y$ by $T(u)=T_{1}(u) \otimes T_{2}(u), u \in$ $X \otimes_{\gamma} Y$. Let $S$ be a completely continuous additive self mapping on $P \otimes Q$ such that $S$ and $T$ commute on $P \otimes Q$. Suppose for every $\sum_{i} p_{i} \otimes q_{i}$ in $P \otimes Q$, there exists one and only one solution $\left(\sum_{i} p_{i} \otimes q_{i}\right)^{0}$ in $P \otimes Q$ of the equation

$$
\begin{aligned}
& \sum_{i} a_{i} \otimes b_{i}=T\left(\sum_{i} a_{i} \otimes b_{i}\right)+T\left(\sum_{i} p_{i} \otimes q_{i}\right)+\sum_{i} p_{i} \otimes q_{i} \\
& \text { where } \sum_{i} a_{i} \otimes b_{i} \in X \otimes_{\gamma} Y
\end{aligned}
$$

Then $(T+T S+S)$ has a fixed point in $P \otimes Q$. 
Proof. We define $J: P \otimes Q \rightarrow P \otimes Q$ by $J\left(\sum_{i} p_{i} \otimes q_{i}\right)=\left(\sum_{i} p_{i} \otimes q_{i}\right)^{0}$. First we show that $J$ is continuous. Let $\left\{\sum_{i} p_{i_{n}} \otimes q_{i_{n}}\right\}_{n}$ be a sequence in $P \otimes Q$ such that

$$
\begin{aligned}
\sum_{i} p_{i_{n}} \otimes q_{i_{n}} \rightarrow & \sum_{i} p_{i} \otimes q_{i} \quad \text { as } \quad n \rightarrow \infty . \text { Now } \\
J\left(\sum_{i} p_{i_{n}} \otimes q_{i_{n}}\right)= & T\left(\sum_{i} p_{i_{n}} \otimes q_{i_{n}}\right)^{0}+T\left(\sum_{i} p_{i_{n}} \otimes q_{i_{n}}\right) \\
& +\sum_{i} p_{i_{n}} \otimes q_{i_{n}} \quad[b y(1)] \\
\lim _{n \rightarrow \infty} J\left(\sum_{i} p_{i_{n}} \otimes q_{i_{n}}\right)= & T\left(\lim _{n \rightarrow \infty}\left(\sum_{i} p_{i_{n}} \otimes q_{i_{n}}\right)^{0}\right)+T\left(\lim _{n \rightarrow \infty} \sum_{i} p_{i_{n}} \otimes q_{i_{n}}\right) \\
& +\sum_{i} p_{i} \otimes q_{i} \\
= & T\left(\lim _{n \rightarrow \infty} J\left(\sum_{i} p_{i_{n}} \otimes q_{i_{n}}\right)\right)+T\left(\sum_{i} p_{i} \otimes q_{i}\right)+\sum_{i} p_{i} \otimes q_{i} .
\end{aligned}
$$

So, $\lim _{n \rightarrow \infty} J\left(\sum_{i} p_{i_{n}} \otimes q_{i_{n}}\right)$ is a solution of the equation (1) . Therefore,

$$
\lim _{n \rightarrow \infty} J\left(\sum_{i} p_{i_{n}} \otimes q_{i_{n}}\right)=\left(\sum_{i} p_{i} \otimes q_{i}\right)^{0}=J\left(\sum_{i} p_{i} \otimes q_{i}\right)
$$

showing that $J$ is continuous.

For $u \in P \otimes Q, J u=u^{0}=T\left(u^{0}\right)+T(u)+u$, using (1). Now,

$$
S(J u)=S\left(u^{0}\right)=S\left(T\left(u^{0}\right)+T(u)+u\right)=T\left(S\left(u^{0}\right)\right)+T(S(u))+S(u),
$$

showing that $S\left(u^{0}\right)$ is a solution of the equation (1) for $S(u)$ in $P \otimes Q$. Hence

$$
S\left(u^{0}\right)=(S(u))^{0} \text { i.e. } S(J u)=J(S u) .
$$

Thus $S$ and $J$ commute. Now we define

$$
K: P \otimes Q \rightarrow P \otimes Q
$$

by $K(u)=S\left(u^{0}\right)=S(J u)$, for $u \in P \otimes Q$.

Since $J$ is continuous and $S$ is completely continuous so the mapping $K$ is completely continuous. Again, since $P$ and $Q$ are bounded subsets, so, $P \otimes Q$ is bounded subset of $X \otimes_{\gamma} Y$. Now, $K^{n}(P \otimes Q)=S^{n} J^{n}(P \otimes Q)$ is bounded for 
$n \in \mathbb{N}$. So, applying Theorem 2.3, we get $K$ has a fixed point, say $\alpha$ in $P \otimes Q$. Therefore,

$$
\begin{aligned}
\alpha=K(\alpha)=S J(\alpha)=J S(\alpha)=(S(\alpha))^{0} & =T\left((S(\alpha))^{0}\right)+T(S(\alpha))+S(\alpha) \\
& =T(\alpha)+T(S(\alpha))+S(\alpha)
\end{aligned}
$$

Thus, $\alpha$ is a fixed point for $T+T S+S$ in $P \otimes Q$.

In Theorem 3.1, instead of taking the bounded subsets $P$ and $Q$, if we take the original space $X \otimes_{\gamma} Y$, the theorem will be as follows:

Theorem 3.2. Let $T_{1}: X \otimes_{\gamma} Y \rightarrow X$ and $T_{2}: X \otimes_{\gamma} Y \rightarrow Y$ be two continuous mappings and $T: X \otimes_{\gamma} Y \rightarrow X \otimes_{\gamma} Y$ be defined by $T(u)=T_{1}(u) \otimes$ $T_{2}(u), u \in X \otimes_{\gamma} Y$. Let $S$ be a completely continuous additive self mapping on $X \otimes_{\gamma} Y$ such that $S T=T S$ and for some $m>1, S^{m}\left(X \otimes_{\gamma} Y\right)$ is bounded. Suppose for every $\sum_{i} x_{i} \otimes y_{i}$ in $X \otimes_{\gamma} Y$, there exists exactly one solution $\left(\sum_{i} x_{i} \otimes\right.$ $\left.y_{i}\right)^{0}$ in $X \otimes_{\gamma} Y$, of the equation

$$
\begin{aligned}
\sum_{i} a_{i} \otimes b_{i}=T\left(\sum_{i} a_{i} \otimes b_{i}\right)+T\left(\sum_{i} x_{i} \otimes y_{i}\right)+\sum_{i} x_{i} \otimes y_{i} & \\
& \sum_{i} a_{i} \otimes b_{i} \in X \otimes_{\gamma} Y .
\end{aligned}
$$

Then $(T+T S+S)$ has a fixed point in $X \otimes_{\gamma} Y$.

Next, we consider $\left(T_{1}, T_{2}\right)$ as a pair of $\left(k, k^{/}\right)$contraction mappings on $P \otimes Q$.

Lemma 3.3. (Refer to [6]) If the pair $\left(T_{1}, T_{2}\right)$ is as defined above (i.e., $\left(k, k^{/}\right)$ contraction), then the mapping $T: P \otimes Q \rightarrow P \otimes Q$ defined by $T(u)=T_{1}(u) \otimes$ $T_{2}(u), u \in P \otimes Q$ has a unique fixed point if $\left(k+k^{/}\right)<1$.

Now, we give an example of the above lemma:

Example 3.4. Let $D_{l^{1} \otimes_{\gamma} \mathbb{K}}$ be a subset of $l^{1} \otimes_{\gamma} \mathbb{K}$ bounded by a constant c. We define $T_{1}: D_{l^{1} \otimes_{\gamma} \mathbb{K}} \rightarrow D_{l^{1}}$ by $T_{1}\left(\sum_{i} a_{i} \otimes x_{i}\right)=\frac{1}{2 c} \sum_{i}\left\{a_{i_{n}} x_{i}\right\}_{n}$, where $a_{i}=\left\{a_{i_{n}}\right\}_{n}$ and $T_{2}: D_{l^{1} \otimes_{\gamma} \mathbb{K}} \rightarrow D_{\mathbb{K}}$ by $T_{2}\left(\sum_{i} a_{i} \otimes x_{i}\right)=\frac{1}{3} \sum_{i}\left\|a_{i}\right\| \cdot\left|x_{i}\right| . \quad\left(D_{l^{1}}\right.$ and $D_{\mathbb{K}}$ are bounded subsets of $l^{1}$ and $\mathbb{K}$ respectively). 
Then $\left(T_{1}, T_{2}\right)$ is a pair of $\left(k, k^{/}\right)$contraction mappings with $\left(k+k^{/}\right)<1$. So the mapping $T: D_{l^{1} \otimes_{\gamma} \mathbb{K}} \rightarrow D_{l^{1} \otimes_{\gamma} \mathbb{K}}$ defined by $T\left(\sum_{i} a_{i} \otimes x_{i}\right)=\frac{1}{6 c} \sum_{i}\left\{M a_{i_{n}} x_{i}\right\}_{n}$, where $M=\left\|a_{i}\right\| \cdot\left|x_{i}\right|$ has a unique fixed point in $D_{l^{1} \otimes_{\gamma} \mathbb{K}}$.

Corollary 3.5. Let $\left(T_{1}, T_{2}\right)$ be a pair of $\left(k, k^{/}\right)$contraction mappings and $T$ be as defined in the Lemma 3.3. Let $S$ be a completely continuous additive self mapping on $P \otimes Q$ such that $S$ commutes with $T$. If $\left(k+k^{/}\right)<1$, then $(T+T S+S)$ has a fixed point in $P \otimes Q$.

Example 3.6. Let $D_{l^{1} \otimes_{\gamma} \mathbb{K}}$ be a subset of $l^{1} \otimes_{\gamma} \mathbb{K}$ bounded by a constant $c$. We define $T_{1}$ and $T_{2}$ as in the Example 3.4, and then we get the pair $\left(T_{1}, T_{2}\right)$ as $\left(k, k^{/}\right)$contraction mappings with $\left(k+k^{/}\right)<1$. Now, $T: D_{l^{1} \otimes_{\gamma} \mathbb{K}} \rightarrow D_{l^{1} \otimes_{\gamma} \mathbb{K}}$ is defined by $T\left(\sum_{i} a_{i} \otimes x_{i}\right)=\frac{1}{6 c} \sum_{i}\left\{M a_{i_{n}} x_{i}\right\}_{n}$, where $M=\left\|a_{i}\right\| \cdot\left|x_{i}\right|$.

Let $S: D_{l^{1} \otimes_{\gamma} \mathbb{K}} \rightarrow D_{l^{1} \otimes_{\gamma} \mathbb{K}}$ be defined by $S\left(\sum_{i} a_{i} \otimes x_{i}\right)=\sum_{i}\left\{\frac{a_{i_{n}} x_{i}}{n}\right\}_{n}$, where $a_{i}=\left\{a_{i_{n}}\right\}_{n}$.

To show that $S$ is compact:

Let $S_{m}: D_{l^{1} \otimes_{\gamma} \mathbb{K}} \rightarrow D_{l^{1} \otimes_{\gamma} \mathbb{K}}$ be defined by

$$
S_{m}\left(\sum_{i} a_{i} \otimes x_{i}\right)=\sum_{i}\left\{a_{i_{1}} x_{i}, \frac{a_{i_{2}} x_{i}}{2}, \frac{a_{i_{3}} x_{i}}{3}, \ldots \ldots, \frac{a_{i_{m}} x_{i}}{m}, 0,0,0, \ldots\right\}
$$

Then each $S_{m}$ is linear, bounded and compact. Also,

$$
\begin{aligned}
\left\|\left(S_{m}-S\right)\left(\sum_{i} a_{i} \otimes x_{i}\right)\right\| & =\left\|\sum_{i}\left\{0,0, \ldots \ldots, 0, \frac{a_{i_{m+1}} x_{i}}{m+1}, \frac{a_{i_{m+2}} x_{i}}{m+2}, \ldots\right\}\right\| \\
& \leqslant \sum_{i} \sum_{j=m+1}^{\infty} \frac{1}{j}\left|a_{i j}\right| \cdot\left|x_{i}\right|<\frac{1}{m+1} \sum_{i} \sum_{j=m+1}^{\infty}\left|a_{i j}\right| \cdot\left|x_{i}\right| \\
& \leqslant \frac{1}{m+1} \sum_{i} \sum_{j=1}^{\infty}\left|a_{i j}\right| \cdot\left|x_{i}\right|=\frac{1}{m+1} \sum_{i}\left\|a_{i}\right\| \cdot\left|x_{i}\right|
\end{aligned}
$$

So, taking the projective tensor norm,

$$
\left\|\left(S_{m}-S\right)\left(\sum_{i} a_{i} \otimes x_{i}\right)\right\|<\frac{1}{m+1}\left\|\sum_{i} a_{i} \otimes x_{i}\right\|
$$

Therefore, $S_{m} \rightarrow S$ and so, $S$ is compact. 
Now, $S$ is additive and clearly, $S$ and $T$ commutes. So, by Corollary 3.5, $(T+T S+S)$ has a fixed point in $D_{l^{1} \otimes_{\gamma} \mathbb{K}}$.

Now we take $P$ and $Q$ as non-empty compact subsets.

Theorem 3.7. Let $P$ and $Q$ be non empty compact subsets of the Banach spaces $X$ and $Y$ respectively. Let $\left(T_{1}, T_{2}\right)$ be a pair of $\left(k, k^{/}\right)$contraction mappings with $\left(k+k^{/}\right)<1$, and the mapping $T$ be as defined earlier. Let $S$ be a continuous self mapping on $P \otimes Q$ such that $T u+T S \alpha+S \alpha \in P \otimes Q$ for all $\alpha, u \in P \otimes Q$. Then $(T+T S+S)$ has fixed point in $P \otimes Q$.

Proof. We fix an arbitrary point $\alpha$ in $P \otimes Q$ and define $J_{\alpha}: P \otimes Q \rightarrow P \otimes Q$ by $J_{\alpha}(u)=T u+T S \alpha+S \alpha, u \in P \otimes Q$. Then for $u_{1}, u_{2} \in P \otimes Q$

$$
\begin{aligned}
\left\|J_{\alpha}\left(u_{1}\right)-J_{\alpha}\left(u_{2}\right)\right\| & =\left\|\left(T u_{1}+T S \alpha+S \alpha\right)-\left(T u_{2}+T S \alpha+S \alpha\right)\right\| \\
& =\left\|T u_{1}-T u_{2}\right\| \leqslant\left(k+k^{\prime}\right)\left\|u_{1}-u_{2}\right\|
\end{aligned}
$$

So, $J_{\alpha}$ is a contraction on $P \otimes Q$ as $\left(k+k^{/}\right)<1$, and has a unique fixed point, say $\hat{\alpha}$ in $P \otimes Q$. So, $\hat{\alpha}=J_{\alpha}(\hat{\alpha})$. Now we define

$$
K: P \otimes Q \rightarrow P \otimes Q
$$

by $K(\alpha)=\hat{\alpha}, \alpha \in P \otimes Q$. For $\alpha, \beta \in P \otimes Q$,

$$
\begin{aligned}
\|K(\alpha)-K(\beta)\| & =\|\hat{\alpha}-\hat{\beta}\| \leqslant\left(k+k^{\prime}\right)\|\hat{\alpha}-\hat{\beta}\|+\left(k+k^{\prime}\right)\|S \alpha-S \beta\| \\
& +\|S \alpha-S \beta\| \\
& \Rightarrow\|\hat{\alpha}-\hat{\beta}\| \leqslant \frac{1+\left(k+k^{\prime}\right)}{1-\left(k+k^{\prime}\right)}\|S \alpha-S \beta\|
\end{aligned}
$$

Since $S$ is continuous, $K$ is also continuous. So by Schauder's Theorem 2.4, $K$ has a fixed point, say $\alpha$, on $P \otimes Q$. Thus,

$$
\alpha=K(\alpha)=\hat{\alpha}=T(\alpha)+T S(\alpha)+S(\alpha)
$$

showing the result.

Theorem 3.8. Let $P$ and $Q$ be two non empty closed subsets of the Banach spaces $X$ and $Y$ respectively. Let $X \otimes_{\gamma} Y$ be reflexive and $\left(T_{1}, T_{2}\right)$ be a pair of $\left(k, k^{\prime}\right)$ contraction mappings on $P \otimes Q$ with $\left(k+k^{\prime}\right)=1$. Let $S$ be a linear continuous self mapping on $P \otimes Q$ such that $(i)\|S\| \leqslant 1$ and (ii) $I-\frac{1}{m}(T+S T+S), m>2, m \in \mathbb{N}$ is demiclosed. Then $\frac{1}{m}(T+S T+S)$ has a fixed point in $P \otimes Q$. 
Proof. Let $\left\{\alpha_{n}\right\}_{n}$ be a sequence in $(0,1)$ such that

$$
\lim _{n \rightarrow \infty} \alpha_{n}=0 .
$$

For $n \in \mathbb{N}$, we define $f_{n}: P \otimes Q \rightarrow P \otimes Q$ by

$$
f_{n}(u)=\frac{1}{m}\left(1-\alpha_{n}\right)(T u+S T u+S u)+\alpha_{n} u_{0}, \text { where } u_{0} \in P \otimes Q .
$$

For $u, v \in P \otimes Q$,

$$
\begin{aligned}
\left\|f_{n}(u)-f_{n}(v)\right\| & \leqslant \frac{1}{m}\left(1-\alpha_{n}\right)[\|T u-T v\|+\|S T u-S T v\|+\|S u-S v\|] \\
& \leqslant \frac{1}{m}\left(1-\alpha_{n}\right)[\|u-v\|+\|S\|\|u-v\|+\|S\|\|u-v\|] \\
& \leqslant \frac{3}{m}\left(1-\alpha_{n}\right)\|u-v\|,(T \text { being nonexpansive })
\end{aligned}
$$

showing that $f_{n}$ is a contraction for $m>2$. Now, $P \otimes Q$ being complete by Banach's contraction mapping principle, $f_{n}$ has a unique fixed point, say $p_{n}$ in $P \otimes Q$. Now,

$$
\begin{aligned}
\left\|p_{n}\right\| & \leqslant \frac{1}{m}\left(1-\alpha_{n}\right)\left[\left\|T p_{n}\right\|+\|S\|\left\|T p_{n}\right\|+\|S\|\left\|p_{n}\right\|\right]+\alpha_{n}\left\|u_{0}\right\| \\
& \leqslant \frac{1}{m}\left(1-\alpha_{n}\right)\left[2\left(\left\|T p_{n}-T u_{0}\right\|+\left\|T u_{0}\right\|\right)+\left\|p_{n}\right\|\right]+\alpha_{n}\left\|u_{0}\right\| \\
& \leqslant \frac{1}{m}\left(1-\alpha_{n}\right)\left[2\left(\left\|p_{n}\right\|+\left\|u_{0}\right\|+\left\|T u_{0}\right\|\right)+\left\|p_{n}\right\|\right]+\alpha_{n}\left\|u_{0}\right\| \\
& =\frac{1}{m}\left(1-\alpha_{n}\right)\left[3\left\|p_{n}\right\|+2\left(\left\|u_{0}\right\|+\left\|T u_{0}\right\|\right)\right]+\alpha_{n}\left\|u_{0}\right\|
\end{aligned}
$$

Dividing both sides by $\left\|p_{n}\right\|$, we get

$$
1 \leqslant \frac{1}{m}\left(1-\alpha_{n}\right)\left[3+2\left(\frac{\left\|u_{0}\right\|}{\left\|p_{n}\right\|}+\frac{\left\|T u_{0}\right\|}{\left\|p_{n}\right\|}\right)\right]+\alpha_{n} \frac{\left\|u_{0}\right\|}{\left\|p_{n}\right\|}
$$

If $\left\{p_{n}\right\}_{n}$ is an unbounded sequence in $P \otimes Q$, then taking $n \rightarrow \infty$, from (2), we get

$$
1 \leqslant \frac{3}{m}\left(1-\alpha_{n}\right) \Rightarrow \frac{m}{3} \leqslant 1-\alpha_{n}<1 \Rightarrow m<3,
$$

a contradiction. So, $\left\{p_{n}\right\}_{n}$ must be bounded. Now, $T$ and $S$ being continuous, are bounded, and so $\left\{T p_{n}\right\}_{n}$ and $\left\{S p_{n}\right\}_{n}$ are bounded. Now,

$$
\left\|p_{n}-\frac{1}{m}\left(T p_{n}+S T p_{n}+S p_{n}\right)\right\| \leqslant \frac{\alpha_{n}}{m}\left[\left\|T p_{n}\right\|+\left\|S T p_{n}\right\|+\left\|S p_{n}\right\|\right]+\alpha_{n}\left\|u_{0}\right\|
$$




$$
\rightarrow 0 \text { as } n \rightarrow \infty
$$

Since $X \otimes_{\gamma} Y$ is reflexive, taking a subsequence if necessary, we may assume that $\left\{p_{n}\right\}_{n}$ has a weak limit (refer to [15]), say $p$. As $I-\frac{1}{m}(T+S T+S$ ) is demiclosed, so, we get

$$
p-\frac{1}{m}(T+S T+S)(p)=0
$$

showing that $p$ is a fixed point for $\frac{1}{m}(T+S T+S), m>2$.

Corollary 3.9. Let $X \otimes_{\gamma} Y$ be reflexive and $\left(T_{1}, T_{2}\right)$ be a pair of $\left(k, k^{/}\right)$ contraction mappings on $P \otimes Q$ with $\left(k+k^{/}\right) \leqslant 1$.

(i) Let $P$ and $Q$ be two non-empty bounded compact subsets of $X$ and $Y$ respectively. If $S$ is a non-expansive self mapping on $P \otimes Q$, such that $I-(T+S T+S)$ is demiclosed then $(T+S T+S)$ has a fixed point in $P \otimes Q$.

Proof. Defining $\left\{\alpha_{n}\right\}_{n}$ as in Theorem 3.8, we define $f_{n}: P \otimes Q \rightarrow P \otimes Q$ by

$$
f_{n}(u)=\left(1-\alpha_{n}\right)(T u+S T u+S u)+\alpha_{n} u_{0}, \text { where } u_{0} \in P \otimes Q .
$$

Then $f_{n}$ is a continuous mapping on $P \otimes Q$ and so, using Theorem $2.4, f_{n}$ has a fixed point say $p_{n}$ in $P \otimes Q$. Since $P \otimes Q$ is bounded, so $\left\{p_{n}\right\}_{n}$ is bounded, and thus $\left\{T p_{n}\right\}_{n}$ and $\left\{S p_{n}\right\}_{n}$ are bounded. So, proceeding as in theorem 3.8, we get $T+S T+S$ has a fixed point in $P \otimes Q$.

(ii) Let $P$ and $Q$ be two non-empty closed and bounded subsets of $X$ and $Y$ respectively. If $S$ is a contraction self mapping with $r \in\left(0, \frac{1}{3}\right]$ on $P \otimes Q$ such that $I-(T+S T+S)$ is demiclosed and $\left(k+k^{\prime}\right) \in\left(0, \frac{1}{2}\right]$ then $(T+S T+S)$ has unique fixed point on $P \otimes Q$.

Proof. Defining $\left\{\alpha_{n}\right\}_{n}$ and $f_{n}$ as above we get, for $u, v \in P \otimes Q$,

$$
\begin{aligned}
\left\|f_{n}(u)-f_{n}(v)\right\| & \leqslant\left(1-\alpha_{n}\right)[\|T u-T v\|+\|S T u-S T v\|+\|S u-S v\|] \\
& \leqslant\left(1-\alpha_{n}\right)\left[\left(k+k^{/}\right)\|u-v\|+r\left(k+k^{/}\right)\|u-v\|+r\|u-v\|\right] \\
& \leqslant\left(1-\alpha_{n}\right)\left[\left(k+k^{/}\right)+r\left(k+k^{/}\right)+r\right]\|u-v\| \\
& \leqslant\left(1-\alpha_{n}\right)\|u-v\| ; \quad \max \left[\left(k+k^{/}\right)+r\left(k+k^{\prime}\right)+r\right] \leqslant 1
\end{aligned}
$$


Then $f_{n}$ is a contraction mapping on $P \otimes Q$ and so, using Theorem 2.5, $f_{n}$ has unique fixed point say $p_{n}$ in $P \otimes Q$. Since $P \otimes Q$ is bounded, so $\left\{p_{n}\right\}_{n}$ is bounded, and thus $\left\{T p_{n}\right\}_{n}$ and $\left\{S p_{n}\right\}_{n}$ are bounded. So, proceeding as in Theorem 3.8, we get $T+S T+S$ has a fixed point on $P \otimes Q$.

(iii) Let $P$ and $Q$ be as in the above Corollary. If $S$ is a linear continuous self mapping on $P \otimes Q$ such that $I-(T+S T+S)$ is demiclosed with $\|S\| \leqslant \frac{1}{3}$ and $\left(k+k^{\prime}\right) \in\left(0, \frac{1}{2}\right]$ then $T+S T+S$ has a unique fixed point in $P \otimes Q$.

Proof. For $\left\{\alpha_{n}\right\}_{n}$ and $f_{n}$ as above we get,

$$
\left\|f_{n}(u)-f_{n}(v)\right\| \leqslant\left(1-\alpha_{n}\right)\|u-v\|, u, v \in P \otimes Q
$$

Rest of the proof follows immediately as above.

In [12], [17] we have the following sufficient conditions for a real sequence for converging to zero.

Lemma 3.10. Let $\left\{\alpha_{n}\right\}_{n}$ be a non-negative real sequence satisfying

$$
\alpha_{n+1} \leqslant\left(1-\gamma_{n}\right) \alpha_{n}+\gamma_{n} \delta_{n}+\sigma_{n}, n=0,1,2, \ldots
$$

If $\left\{\gamma_{n}\right\}_{n=1}^{\infty} \subset(0,1),\left\{\delta_{n}\right\}_{n=1}^{\infty}$ and $\left\{\sigma_{n}\right\}_{n=1}^{\infty}$ satisfy the conditions:

I. $\sum_{n=1}^{\infty} \gamma_{n}=\infty$.

II. either $\lim \sup _{n \rightarrow \infty} \delta_{n} \leqslant 0$ or $\sum_{n=1}^{\infty}\left|\gamma_{n} \delta_{n}\right|<\infty$.

III. $\sum_{n=1}^{\infty}\left|\sigma_{n}\right|<\infty$.

Then $\lim _{n \rightarrow \infty} \alpha_{n}=0$.

In 2013, Songnian He and Wenlong Zhu defined an iteration scheme which was named as boundary point method (refer to [12]). Considering this, here we derive the following iteration scheme:

$$
\begin{aligned}
x_{0} & \in P \otimes Q \\
y_{n} & =\alpha_{n} x_{n}+\left(1-\alpha_{n}\right)\left(T x_{n}+S T x_{n}+S x_{n}\right) \\
x_{n+1} & =\alpha_{n} \lambda_{n} y_{n}+\left(1-\alpha_{n}\right)(T u+S T u+S u)
\end{aligned}
$$


where $u \in P \otimes Q$ is an arbitrary (but fixed) element in $P \otimes Q,\left\{\alpha_{n}\right\}_{n}$ is a sequence in $(0,1)$ and $\left\{\lambda_{n}\right\}_{n}$ is a monotonic increasing sequence in $[0,1]$.

Theorem 3.11. Let $\left\{\alpha_{n}\right\}_{n}$ and $\left\{\lambda_{n}\right\}_{n}$ satisfy the following conditions:

A1. $\frac{\alpha_{n}}{1-\alpha_{n} \lambda_{n}} \rightarrow 0$

A2. $\sum_{n=1}^{\infty}\left(1-\alpha_{n} \lambda_{n}\right)=\infty$.

A3. $\sum_{n=1}^{\infty}\left|\alpha_{n}-\alpha_{n-1}\right|<\infty$.

Then $\left\{x_{n}\right\}_{n}$ of the above iteration scheme converges weakly to the unique fixed point of $(T+S T+S)$ in $P \otimes Q$.

Proof. We have $\left\{x_{n}\right\}_{n},\left\{y_{n}\right\}_{n},\left\{T x_{n}\right\}_{n},\left\{S T x_{n}\right\}_{n}$ and $\left\{S x_{n}\right\}_{n}$ are sequences in the bounded subset $P \otimes Q$ of $X \otimes_{\gamma} Y$.

$$
\left\|x_{n+1}-(T u+S T u+S u)\right\| \leqslant \alpha_{n}\left(\lambda_{n}\left\|y_{n}\right\|+\|T u\|+\|S T u\|+\|S u\|\right) \rightarrow 0
$$

[using A1]

Now we will show that $\left\|x_{n+1}-x_{n}\right\| \rightarrow 0$

$$
\begin{aligned}
& \left\|x_{n+1}-x_{n}\right\|=\| \alpha_{n} \lambda_{n} y_{n}+\left(1-\alpha_{n}\right)(T u+S T u+S u) \\
& \quad-\left[\alpha_{n-1} \lambda_{n-1} y_{n-1}+\left(1-\alpha_{n-1}\right)(T u+S T u+S u)\right] \| \\
& \leqslant \alpha_{n} \lambda_{n}\left\|y_{n}-y_{n-1}\right\|+\left|\alpha_{n}-\alpha_{n-1}\right|\left(\lambda_{n-1}\left\|y_{n-1}\right\|\right) \\
& +\alpha_{n}\left|\lambda_{n}-\lambda_{n-1}\right|\left\|y_{n-1}\right\| \\
& \quad+\left|\alpha_{n}-\alpha_{n-1}\right|[\|T u\|+\|S T u\|+\|S u\|] \\
& \left.\qquad y_{n-y_{n-1}\|\leqslant\| x_{n}-x_{n-1} \|+\left|\alpha_{n}-\alpha_{n-1}\right|\left[\left\|T x_{n-1}\right\|\right.}+\left\|S T x_{n-1}\right\|+\left\|S x_{n-1}\right\|+\left\|x_{n-1}\right\|\right] \\
& \\
& +\left|\alpha_{n}-\alpha_{n-1}\right|[\|T u\| \\
& +\|S T u\|+\|S u\|+\lambda_{n-1}\left\|y_{n-1}\right\| \\
& \left.+\left\|T x_{n-1}\right\|+\left\|S T x_{n-1}\right\|+\left\|S x_{n-1}\right\|+\left\|x_{n-1}\right\|\right]
\end{aligned}
$$


Since, $\sum_{n=1}^{\infty}\left|\lambda_{n}-\lambda_{n-1}\right|<\infty$ as $\left\{\lambda_{n}\right\}_{n} \subset[0,1]$ is a monotonic increasing sequence and by the conditions $A 1, A 2$ and $A 3$ we will easily get according to the lemma $3.10\left\|x_{n+1}-x_{n}\right\| \rightarrow 0$. Hence,

$$
\begin{aligned}
\left\|x_{n}-\left(T x_{n}+S T x_{n}+S x_{n}\right)\right\| \leqslant & \left\|x_{n+1}-x_{n}\right\|+\left\|x_{n+1}-(T u+S T u+S u)\right\| \\
& +\left\|(T u+S T u+S u)-\left(T x_{n}+S T x_{n}+S x_{n}\right)\right\| \\
& \leqslant\left\|x_{n+1}-x_{n}\right\|+\left\|x_{n+1}-(T u+S T u+S u)\right\| \\
& +\left\|u-x_{n}\right\|
\end{aligned}
$$

Since $\left\{x_{n}\right\}_{n}$ is a Cauchy sequence so, it converges to say, $p$. Without loss of generality, we can take $u=p$ here so that $\left\|u-x_{n}\right\| \rightarrow 0$. Thus

$$
\left\|x_{n}-\left(T x_{n}+S T x_{n}+S x_{n}\right)\right\| \rightarrow 0
$$

Since $I-(T+S T+S)$ is demiclosed so proceeding Theorem 3.8 we have the iteration scheme converges weakly to the unique fixed point in $P \otimes Q$.

\section{Concluding Remarks}

In [15], J. Penot discussed about asymptotically contractive maps on a subset $C$ of a Banach space $X$. The map $f: C \rightarrow X$ is called asymptotically contractive on $C$ if there exists some $x_{0} \in C$ such that

$$
\lim _{x \in C} \sup _{\|x\| \rightarrow \infty} \frac{\left\|f(x)-f\left(x_{0}\right)\right\|}{\left\|x-x_{0}\right\|}<1
$$

Here the following problem arises:

Can we obtain analogous result as in Theorem 3.1 or 3.7 taking $S$ as asymptotically contractive map on $P \otimes Q ?$

\section{References}

[1] S. Banach, Sur les operations dans les ensembles abstraits et leur application aux equations integrales, Fund. Math. 3, No. 1 (1922), 133-181

[2] F. F. Bonsal, and J. Duncan, Complete Normed algebras, Springer-Verlag, Berlin Heidelberg New York, (1973)

[3] Felix. E. Browder, On a Generalization of the Schauder Fixed Point Theorem, Duke Mathematical Journal, 26(2), (1959), 291-303 
[4] G.L. (JR.) Cain, and M.Z. Nashed Fixed points and stability for a sum of two operators in locally convex spaces, Pacific J. Math. 39, (1971), 581-592

[5] B. C. Dhage, Remarks on Two Fixed-Point Theorems Involving the Sum and the Product of Two Operators,Computers and Mathematics with Applications, 46, (2003), 1779-1785

[6] D. Das and N. Goswami Some fixed point theorems on tensor product spaces, Proceedings of ICFM 2015, Gauhati University, Guwahati, Assam, India

[7] D. Das, N. Goswami, Fixed Points of Different Contractive Type Mappings on Tensor Product Spaces, IJIRSET, 3 No.7 (2014)

[8] D. Das, N. Goswami,Fixed Points of Mapping Satisfying a Weakly Contractive Type Condition, Journal of Math. Res. with Appl., 36, No. 1 (2016), 70-78

[9] D.E. Edmunds, Remarks on nonlinear functional equations, Math. Ann. 174, (1967), 233-239

[10] Olga Hadzic, Some Fixed Point Theorems in Banach Spaces, Review of Research Faculty of Science, 8, (1978), 13-19

[11] Olga Hadzic, A Fixed Point Theorem For The Sum of Two Mappings, Proc. Amer. Math. Soc. 85, No. 1 (1982), 37-41

[12] Songnian He, and Wenlong Zhu, A Modified Mann Iteration by Boundary Point Method for Finding Minimum-Norm Fixed Point of Nonexpansive Mappings, Hindawi Publishing Corporation. 2013, (2013), 6 pages

[13] M.A. Krasnoselskii, Two remarks on the method of successive approximations, Uspehi Math. Nauk. 10,(1955), 123-127

[14] M.A. Nashed, and J.S.W. Wong, Some variants of a fixed point theorem Krasnoselskii and applications to nonlinear integral equations, J. Math. Mech. 18, (1969), 767-777

[15] Penot Jean-Paul A Fixed-Point Theorem For Asymptotically Contractive Mapping, Proc. Amer. Math. Soc., 131, No.8 (2003), 2371-2377

[16] J. Reinermann, Fixpunkts.tze vom Krasnoselskii-Typ., Math. Z. 119, (1971), 339-344

[17] Rodrigo Lpez Pouso Schauders fixed-point theorem: new applications and a new version for discontinuous operators, Boundary Value Problems, (2012), 1-14

[18] V.M. Sehgal, and S.P. Singh, A fixed point theorem for the sum of two mappings, Math. Japonica 23, (1978), 71-75

[19] P. Vijayraju, Fixed Point Theorems For a Sum of Two Mappings in Locally Convex Spaces, Internat. J. Math. and Math. Sci., 17, No.4 (1994), 681-686,

[20] F. Wang, and H. K. Xu, Approximating curve and strong convergence of the CQ algorithm for the split feasibility problem, Journal of Inequalities and Applications, (2010), 13 pages. 
